Supporting Information

\title{
Transparent Graphene/BN-Graphene Stacked Nanofilms for Electrocatalytic Oxygen Evolution
}

\author{
Rajib Paul ${ }^{1 *}$, Min Wang1‡, Ajit Roy ${ }^{2}$ \\ ${ }^{1}$ Department of Macromolecular Science and Engineering, Case School of Engineering, Case Western Reserve \\ University, 10900 Euclid Avenue, Cleveland, Ohio 44106, United States. \\ ${ }^{2}$ Materials and Manufacturing Directorate, Air Force Research Laboratory, Wright-Patterson AFB, OH 45433, \\ United States.
}

‡Present address: National Renewable Energy Laboratory, 15257 Denver West Parkway, Golden, C0 80401, United States.

*Corresponding Author, Email: rxp348@case.edu 


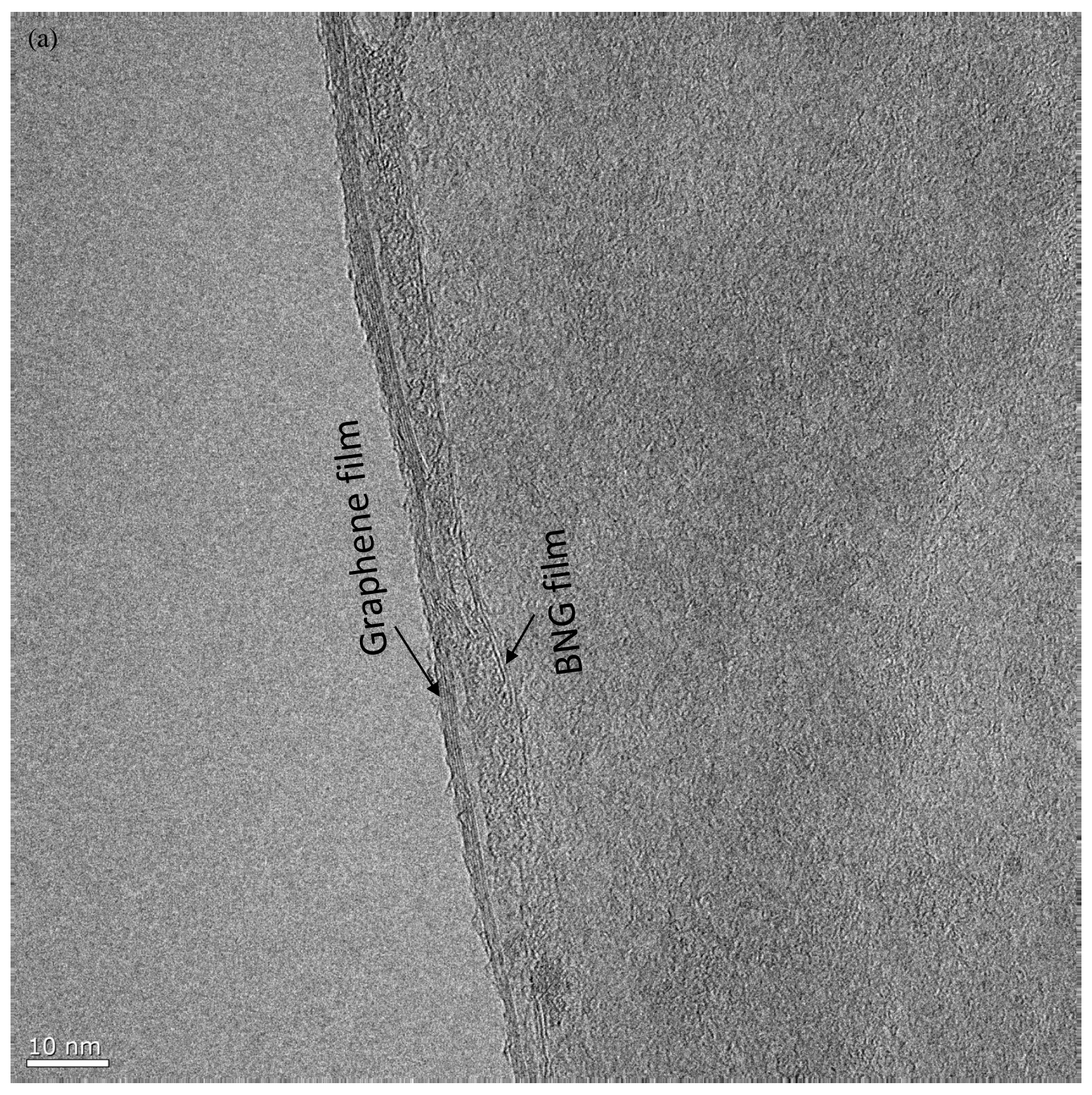




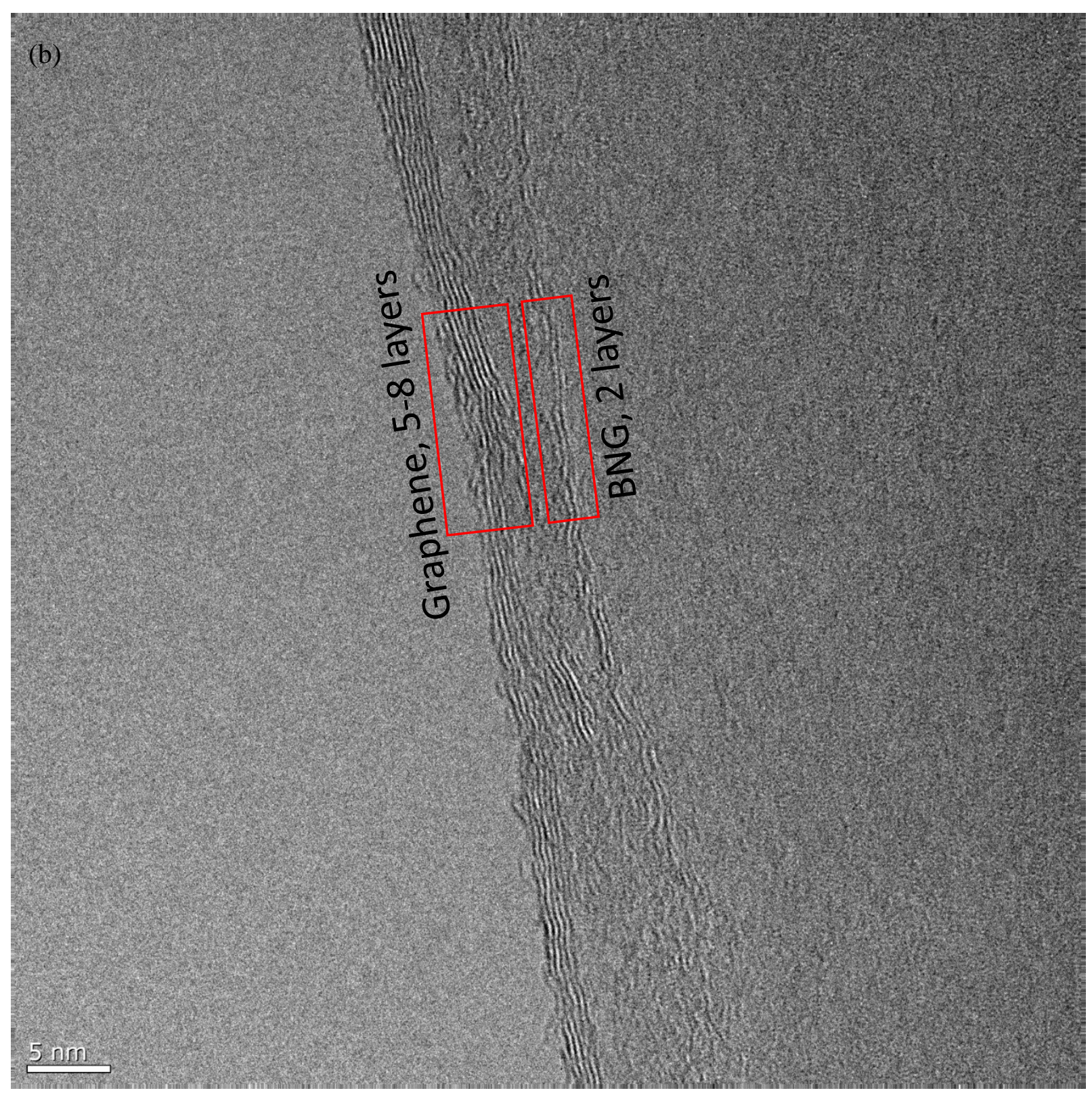

Figure S1 (a, b). Representative TEM images of stacked G-BNG film with different magnifications. 


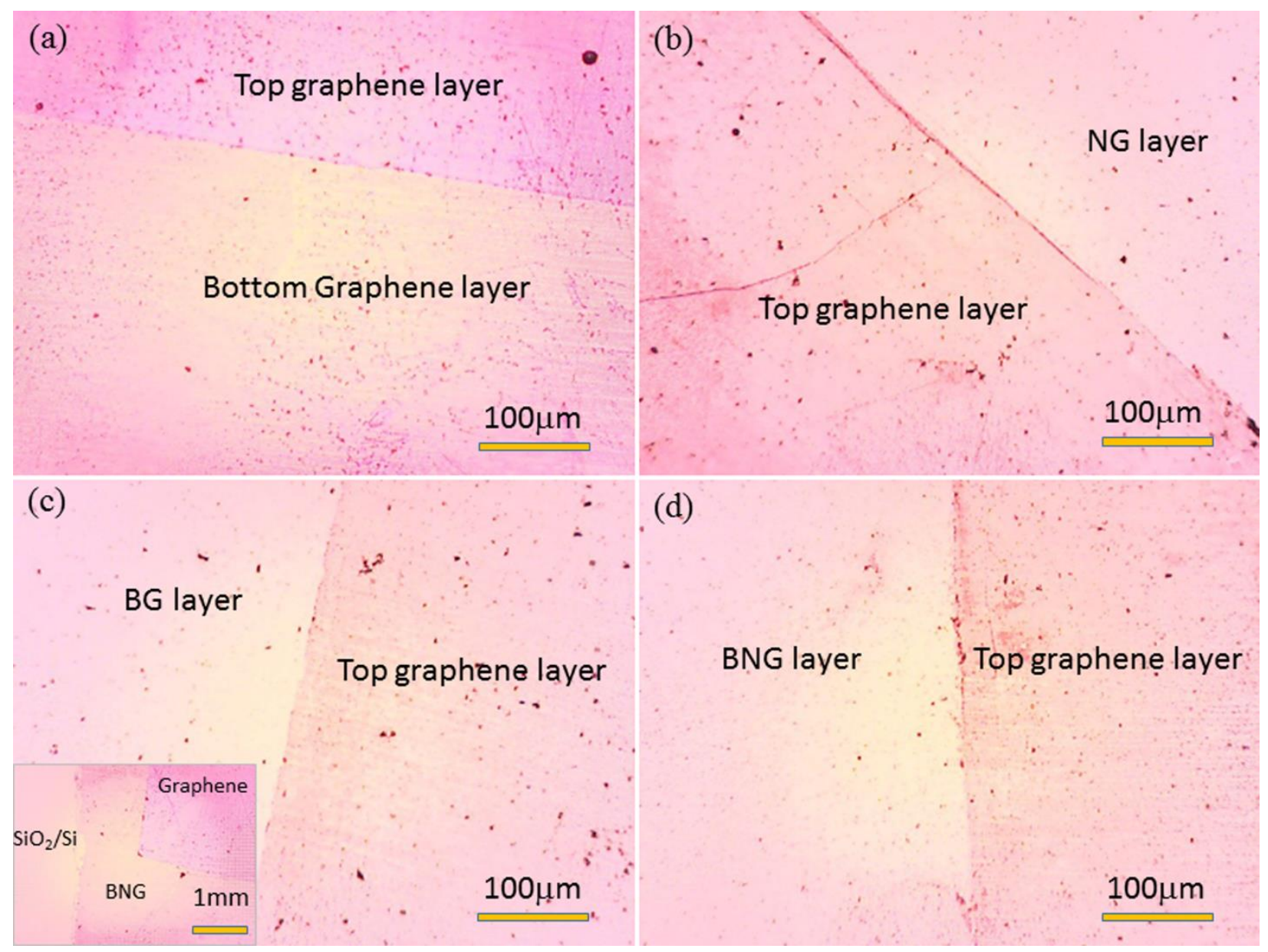

Figure S2. Optical images of stacked double layer for (a) G-G, (b) G-NG, (c) G-BG and (d) GBNG films. Inset of (c) shows G-BNG film at large scale. 

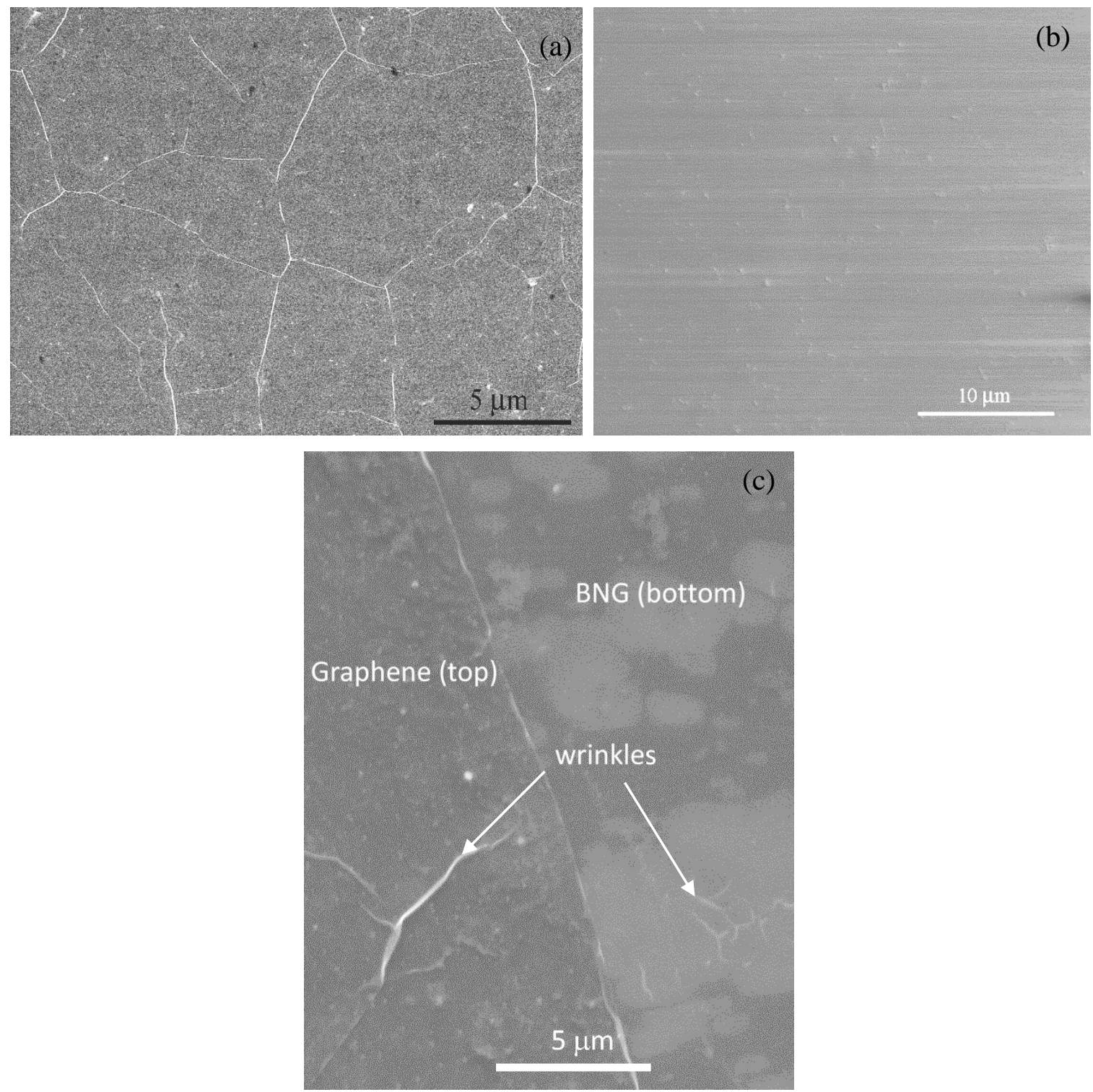

Figure S3. Representative SEM images of (a) graphene on $\mathrm{Cu}$ foil (the grain boundaries of $\mathrm{Cu}$ are visible through the graphene layer as white lines), (b) $\mathrm{BNG}$ film on $\mathrm{SiO}_{2} / \mathrm{Si}$ substrate and (c) G-BNG double layer stacked nanofilm on glass substrate. 

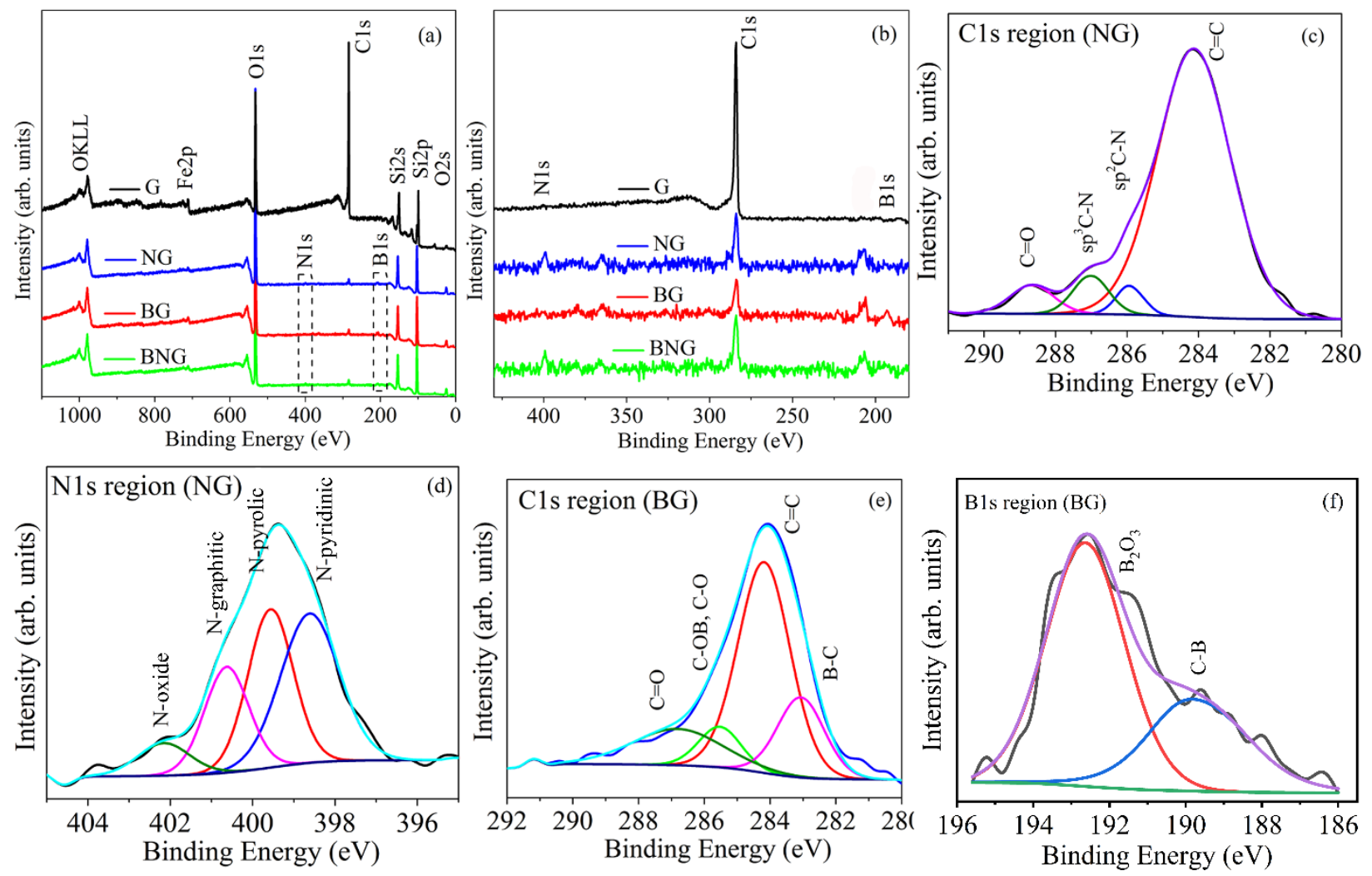

Figure S4. X-ray photoelectron spectra of different samples (a) full range survey spectra, (b) survey spectra showing B 1s, C 1s and N 1s regions (c) high resolution (HR) C1S region for NG, (d) HR N1s region for NG, (e) HR C1s region for BG, (f) HR B1s region for BG.

In Fig S4(a), the intensity of C 1s, N 1s and B 1s spectra for NG, BG and BNG films are weak due to very thin layer thickness as evidenced from TEM imaging as well as the strong Si $2 \mathrm{p}$ and O 1s peaks arising mainly from substrate. Careful analysis of the peaks reveals that the atomic content ratio of $\mathrm{Si}$ to $\mathrm{O}$ element is almost 1:2 which confirms that these peaks are originated from the substrate $\left(\mathrm{SiO}_{2} / \mathrm{Si}\right)$. Deconvoluted C $1 \mathrm{~s}$ and B $1 \mathrm{~s}$ spectra for BG film have been presented in Fig. $\mathrm{S} 4$ (e) and (f) respectively. The peak at $283.1 \mathrm{eV}$ can be attributed to $\mathrm{CB}$ bonds (16.7 at. \%) while the other peaks can be correlated to $\mathrm{C}=\mathrm{C}$ and oxygen containing bonds with $\mathrm{C}$ atoms. ${ }^{\text {S1-S2 }}$ The $\mathrm{B}$ 1s spectrum can be divided into two sub-peaks related to C-B (at $190.3 \mathrm{eV}$ and 4.02 at. \%) and $\mathrm{B}_{2} \mathrm{O}_{3}$ (at $192.7 \mathrm{eV}$ and 8.16 at. \%) bonds. ${ }^{\mathrm{S} 1, \mathrm{~S} 3}$ 
Table S1: Chemical composition of different films obtained from XPS analysis.

\begin{tabular}{|c|c|c|c|c|c|c|c|c|c|}
\hline \multirow[t]{2}{*}{ Sample } & \multirow{2}{*}{$\begin{array}{l}\text { C \% } \\
\text { (at.) }\end{array}$} & \multicolumn{5}{|c|}{$\mathrm{N} \%$ (at.) } & \multicolumn{3}{|c|}{ B \% (at.) } \\
\hline & & N-B & $\begin{array}{c}\text { N- } \\
\text { Pyridinic }\end{array}$ & $\begin{array}{c}\text { N- } \\
\text { Pyrolic }\end{array}$ & $\begin{array}{c}\text { N- } \\
\text { Graphitic }\end{array}$ & $\begin{array}{c}\text { N- } \\
\text { Oxide }\end{array}$ & B-N & B-C & $\mathrm{B}_{2} \mathrm{O}_{3}$ \\
\hline $\mathrm{NG}$ & 91.5 & - & 3.33 & 2.71 & 1.78 & 0.64 & - & & \\
\hline $\mathrm{BG}$ & 87.9 & - & & & & & - & 4.02 & 8.16 \\
\hline $\mathrm{BNG}$ & 79.5 & 0.77 & 1.66 & 3.36 & 2.91 & 0.94 & 4.62 & 3.04 & 3.22 \\
\hline
\end{tabular}




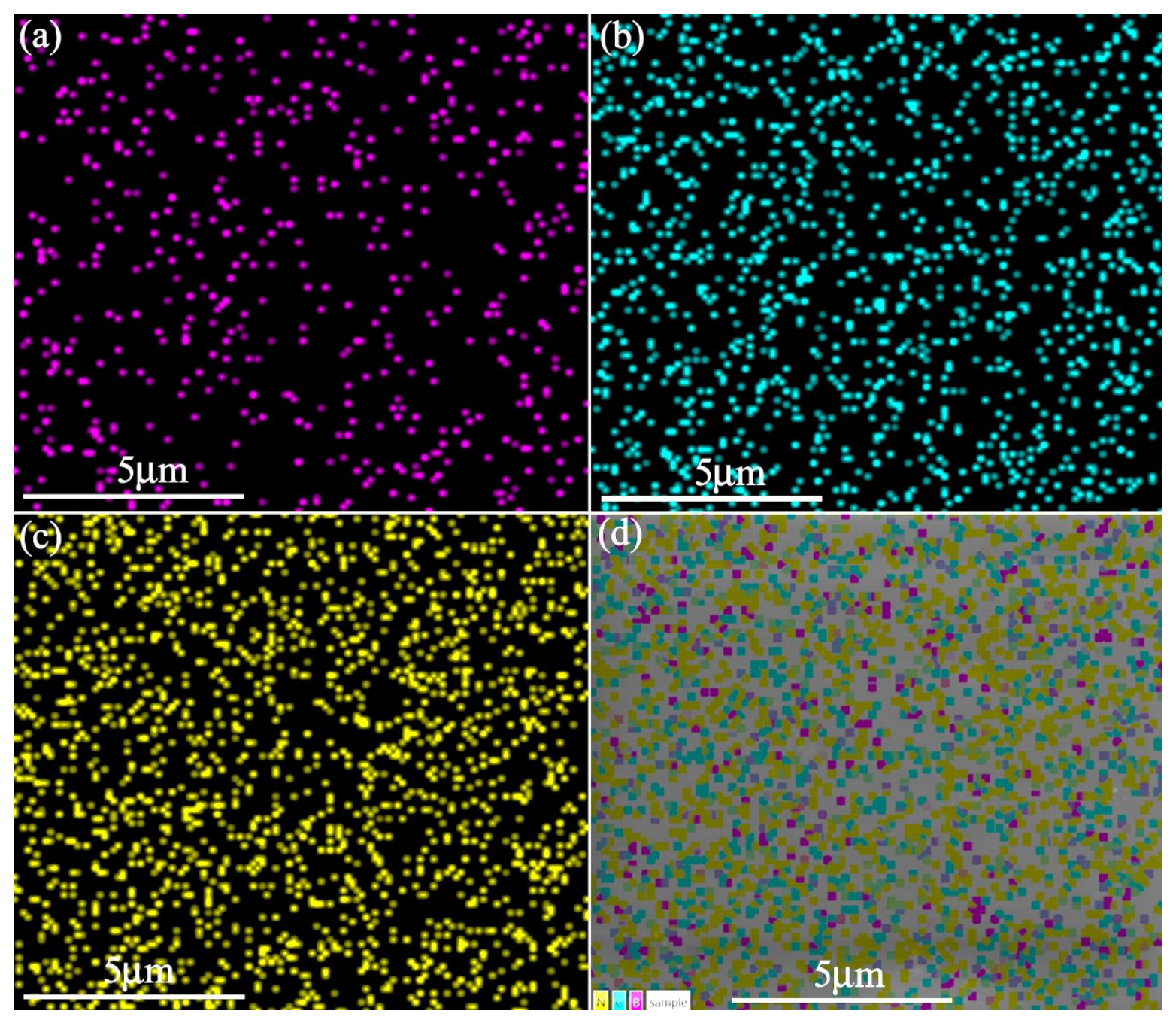

Figure S5. EDS elemental mapping of (a) B, (b) C, (c) N and (d) B, C and N all together on BNG film. 

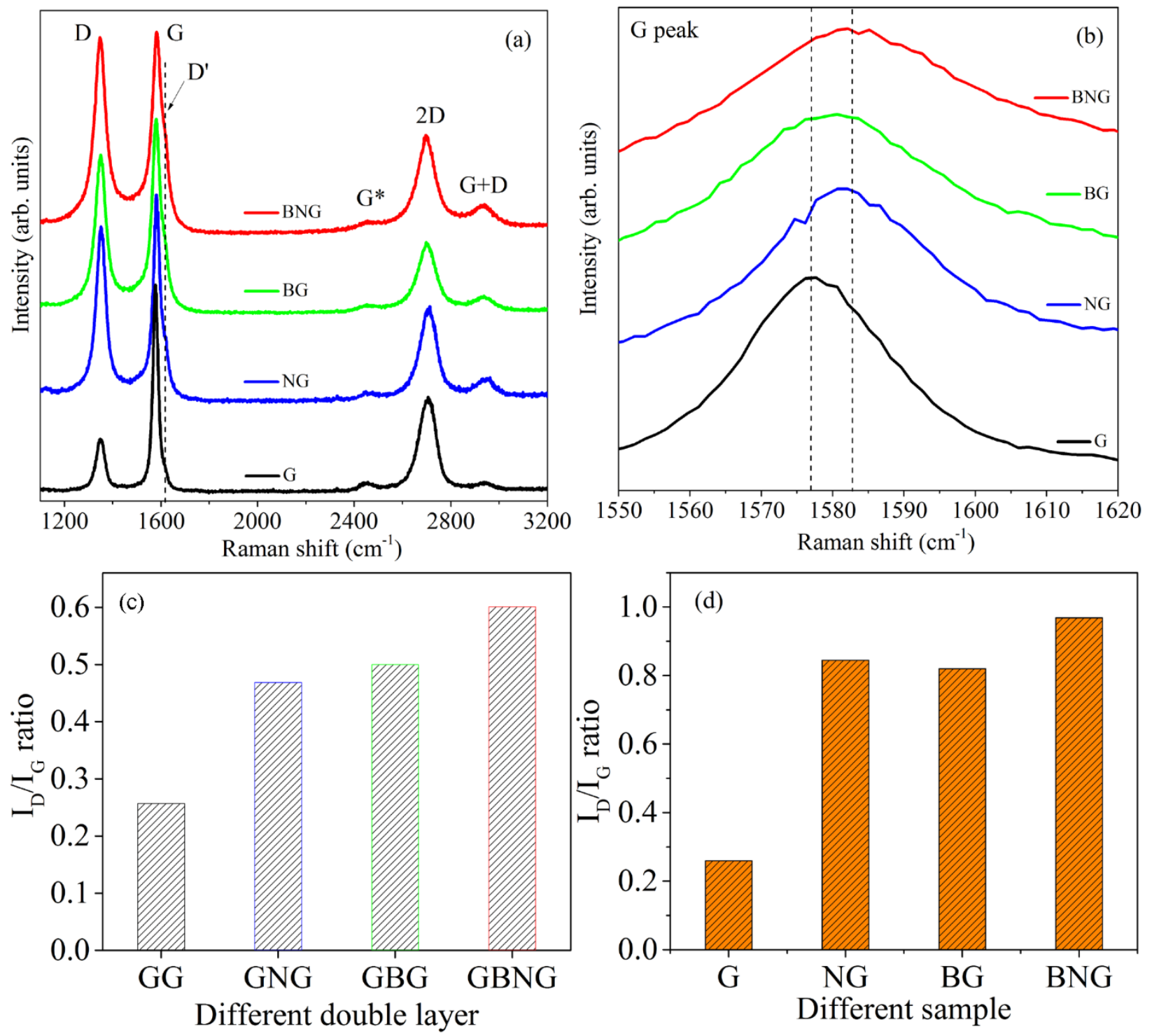

Figure S6. (a) Raman spectra of different single layer films, (b) G peak positions for single layer films (c) $I_{D} / I_{G}$ ratio for different double layer films and (d) $I_{D} / I_{G}$ ratio for different one layer films.

For Raman measurement on double layer films, we need to be sure about the fact that the laser penetrates to the bottom layer during Raman spectra recording on the double layer films. For this purpose, we have opted a theoretical approach to estimate the penetration depth of laser into graphene lattice. As the Raman intensity is generally proportional to the thickness of graphene layers, the penetration depth (L) of the 514 $\mathrm{nm}$ laser used for Raman measurements can be estimated according to the Beer-Lambert law, I/ $\mathrm{I}_{0}=\mathrm{e}^{-\alpha \mathrm{L}}$, where $\alpha=4 \pi \mathrm{k} / \lambda$. Here $\lambda$ is the wavelength of laser= $514 \mathrm{~nm}$ and $\mathrm{k}=1.3$, is the extinction coefficient of graphite. ${ }^{\mathrm{S} 4}$ Using these values $\alpha=0.0318$ and the penetration depth is calculated to be $32 \mathrm{~nm}$ for graphite which corresponds to 94 graphene monolayers. Therefor the Raman spectra we have obtained on double layer films corresponds to the overall double layers as the film thickness is around 6-7 layers as observed in TEM images. 


\section{Tauc's analysis for band gap calculation}

Tauc's equation for band gap calculation can be expressed by, ${ }^{\mathrm{S} 5}$

$\omega^{2} \epsilon=\left(h \omega-E_{g}\right)^{2}$

where $\varepsilon$ is the complex part of the dielectric function which is proportional to the optical absorbance according to Tauc. $\omega$ is the angular frequency of the incident radiation. According to the technique, the plot of $\varepsilon^{0.5} / \lambda$ versus $1 / \lambda$ is a straight line and the intersection point with the $x$ axis is $1 / \lambda_{\mathrm{g}}\left(\lambda_{\mathrm{g}}\right.$ is the gap wavelength). The optical band gap is then calculated based on $E_{\mathrm{g}}=\mathrm{hc} / \lambda_{\mathrm{g}}$ For simplicity in calculation, we have plotted $\varepsilon^{0.5} / \lambda$ versus photonic energy (eV) as shown in Fig. 58 .

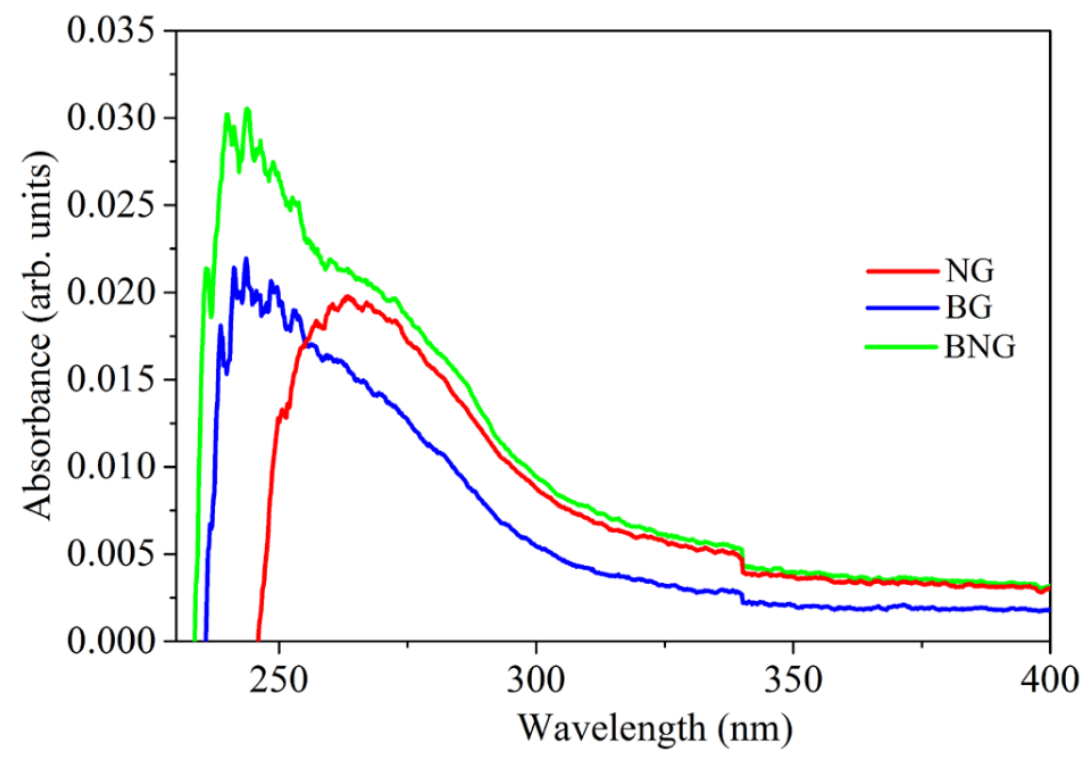

Figure S7. Optical absorbance spectra for different doped films (NG, BG and BNG films). 

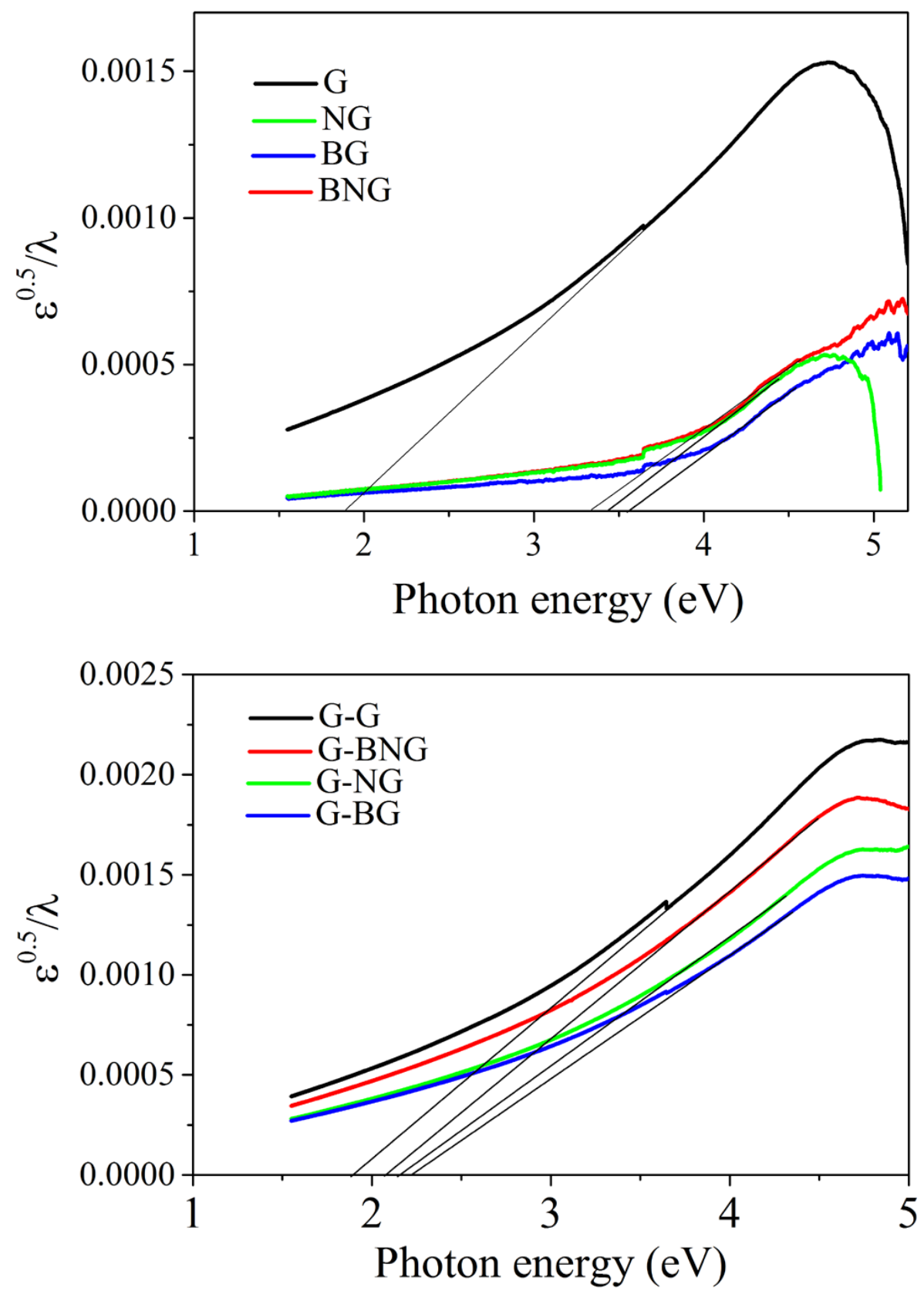

Figure S8. Tauc plot for different single layer ( $\mathrm{G}, \mathrm{NG}, \mathrm{BG}$ and $\mathrm{BNG})$ and stacked double layer (G-G, G-NG, G-BG and G-BNG) films. 
Table S2: Photonic Bandgap obtained from optical absorbance spectra using Tauc's equation

\begin{tabular}{|c|c|c|}
\hline Film & $\begin{array}{l}\text { Photonic Bandgap } \\
\text { (eV) }\end{array}$ & Explanation \\
\hline G & 1.90 & $\pi-\pi *$ excitation \\
\hline NG & 3.45 & $\mathrm{~N}$ doping \\
\hline BG & 3.58 & B doping \\
\hline BNG & 3.34 & BN doping \\
\hline G-G & 1.89 & $\pi-\pi *$ excitation \\
\hline G-NG & 2.22 & $\begin{array}{c}\text { Charge transfer } \\
\text { and } \pi-\pi * \\
\text { excitation }\end{array}$ \\
\hline G-BG & 2.16 & $\begin{array}{c}\text { Charge transfer } \\
\text { and } \pi-\pi * \\
\text { excitation }\end{array}$ \\
\hline G-BNG & 2.09 & $\begin{array}{c}\text { Charge transfer } \\
\text { and } \pi-\pi * \\
\text { excitation }\end{array}$ \\
\hline
\end{tabular}



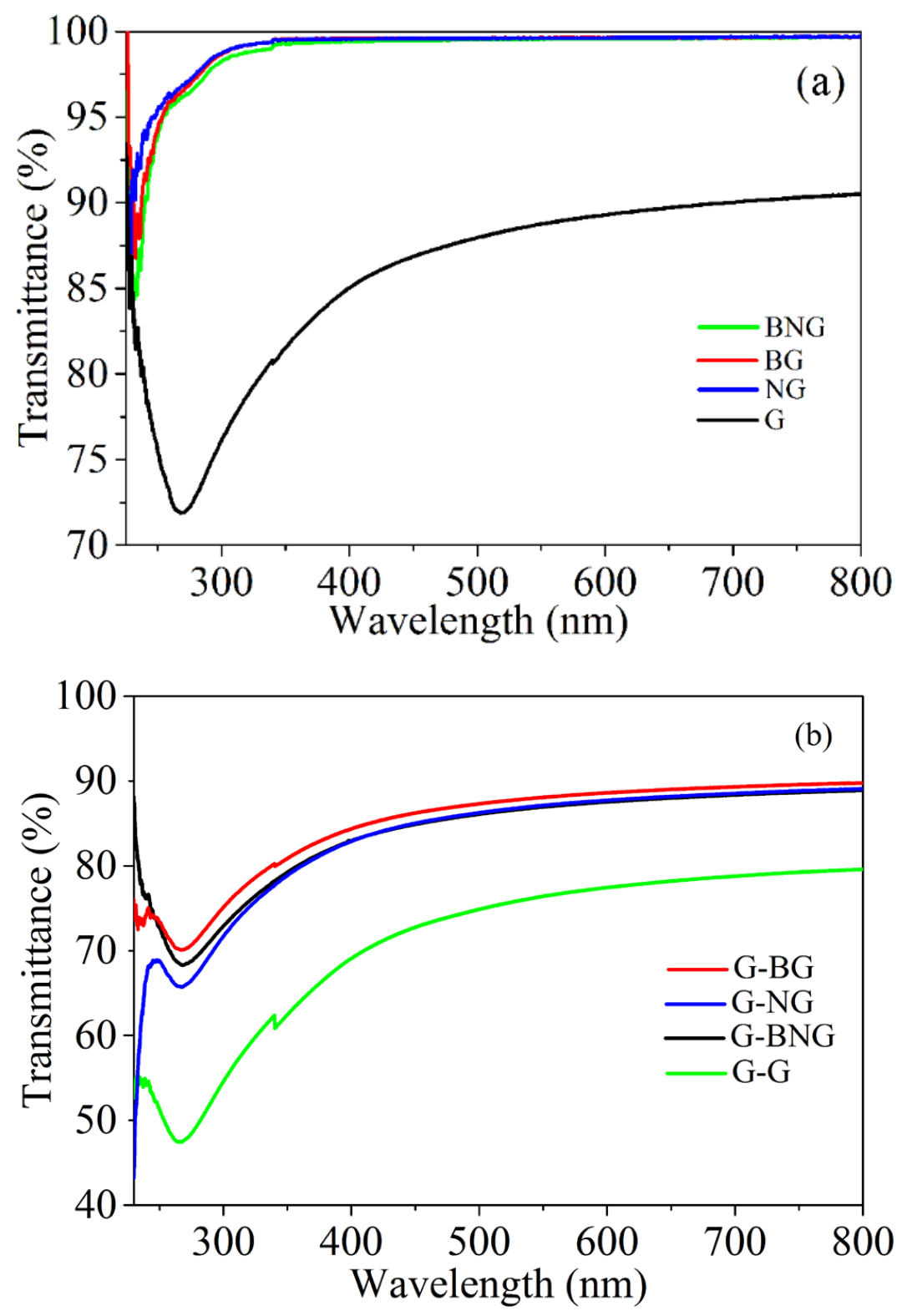

Figure S9. Optical transmittance spectra for different (a) single layer films and (b) double layer films. 

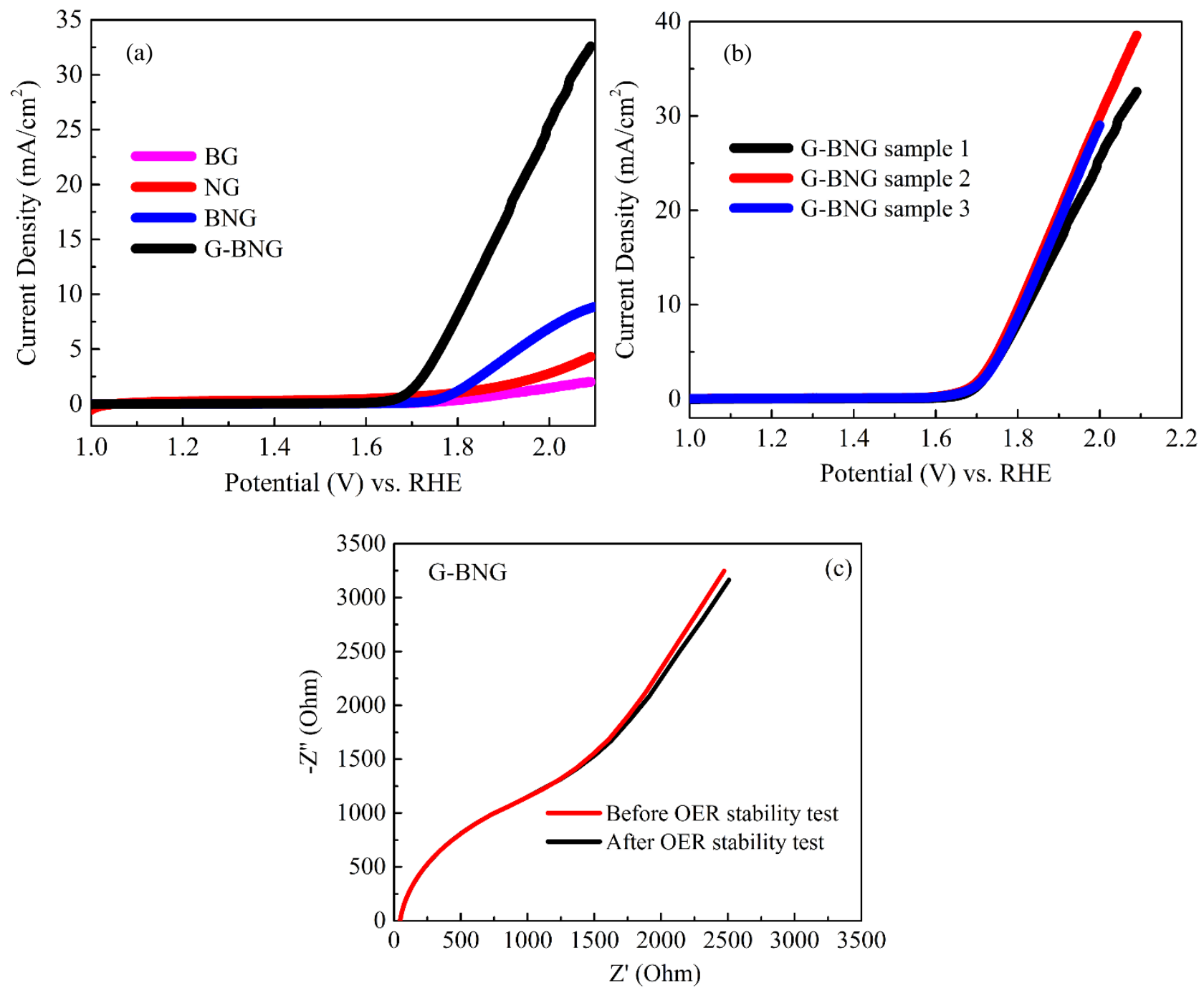

Figure S10. (a) OER polarization curves of BG, NG, BNG and G-BNG at a scan rate of $10 \mathrm{mV} / \mathrm{s}$ in $\mathrm{O}_{2}$ saturated $0.1 \mathrm{M} \mathrm{KOH}$ electrolyte; (b) Reproducible OER polarization curves of three G-BNG samples at a scan rate of $10 \mathrm{mV} / \mathrm{s}$; (c) Impendence spectra of G-BNG film before and after the OER stability test. 
Table S3. Comparison of OER performance of double layer films with reported heteroatom doped 2D or 3D materials

\begin{tabular}{|c|c|c|c|c|c|c|c|c|}
\hline Material & Method & $\begin{array}{l}\text { Mass } \\
\text { loading } \\
(\mathrm{mg} / \mathrm{cm} \\
\left.{ }^{2}\right)\end{array}$ & $\begin{array}{l}\text { Electro } \\
\text { lyte }\end{array}$ & $\begin{array}{l}\text { Onset } \\
\text { potential } \\
\text { (V vs } \\
\text { RHE) }\end{array}$ & $\begin{array}{l}\text { Potential } \\
\text { at } 10 \\
\mathbf{m A} / \mathbf{c m}^{2} \\
\text { (V vs } \\
\text { RHE) }\end{array}$ & $\begin{array}{l}\text { Tafel } \\
\text { slope } \\
\text { (mV/de } \\
\text { c) }\end{array}$ & $\begin{array}{l}\text { Retentivity } \\
\text { (\%), scan } \\
\text { rate }\end{array}$ & Ref. \\
\hline G-BNG & $\begin{array}{l}\text { CVD- } \\
\text { Chemical }\end{array}$ & $\begin{array}{l}0.000 \\
68^{*}\end{array}$ & $\begin{array}{l}0.1 \mathrm{M} \\
\mathrm{KOH}\end{array}$ & 1.60 & 1.81 & 143.22 & $\begin{array}{l}81.6 \text { after } 14 \\
\mathrm{~h}, 10 \mathrm{mV} / \mathrm{s}\end{array}$ & $\begin{array}{l}\text { This } \\
\text { wor } \\
\mathrm{k}\end{array}$ \\
\hline G-BG & $\begin{array}{l}\text { CVD- } \\
\text { Chemical }\end{array}$ & $\begin{array}{l}\sim 0.000 \\
68\end{array}$ & $\begin{array}{l}0.1 \mathrm{M} \\
\mathrm{KOH}\end{array}$ & 1.66 & 1.85 & 191.97 & - & $\begin{array}{l}\text { This } \\
\text { wor } \\
\mathrm{k}\end{array}$ \\
\hline G-NG & $\begin{array}{l}\text { CVD- } \\
\text { Chemical }\end{array}$ & $\begin{array}{l}\sim 0.000 \\
68\end{array}$ & $\begin{array}{l}0.1 \mathrm{M} \\
\mathrm{KOH}\end{array}$ & 1.67 & 1.87 & 231.87 & - & $\begin{array}{l}\text { This } \\
\text { wor } \\
\mathrm{k}\end{array}$ \\
\hline $\begin{array}{l}\mathrm{N}- \\
\text { graphene }\end{array}$ & $\begin{array}{l}\text { Chemical+ } \\
\text { hydrotherm } \\
\text { al+pypolys } \\
\text { is }\end{array}$ & 0.243 & $\begin{array}{l}0.1 \mathrm{M} \\
\mathrm{KOH}\end{array}$ & 1.67 & $\begin{array}{l}2.0 \text { at } 3 \\
\mathrm{~mA} / \mathrm{cm}^{2}\end{array}$ & - & - & S6 \\
\hline $\begin{array}{l}\text { BN-C } \\
\text { hollow } \\
\text { sphere }\end{array}$ & Chemical & - & $\begin{array}{l}0.1 \mathrm{M} \\
\mathrm{KOH}\end{array}$ & - & - & 277.1 & - & S7 \\
\hline $\begin{array}{l}\mathrm{CoO}_{3} / \mathrm{BN} \\
-\mathrm{C} \\
\text { hollow } \\
\text { sphere }\end{array}$ & Chemical & - & $\begin{array}{l}0.1 \mathrm{M} \\
\mathrm{KOH}\end{array}$ & 1.6 & 1.72 & 63.0 & - & S7 \\
\hline N,S-GO & $\begin{array}{l}\text { Solid state } \\
\text { thermolysis }\end{array}$ & 0.528 & $\begin{array}{l}0.1 \mathrm{M} \\
\mathrm{KOH}\end{array}$ & $\sim 1.7$ & - & - & - & S8 \\
\hline $\begin{array}{l}\text { B- } \\
\text { MWNT }\end{array}$ & $\begin{array}{l}\text { Chemical+ } \\
\text { Pyrolysis }\end{array}$ & 0.06 & $\begin{array}{l}0.1 \mathrm{M} \\
\mathrm{KOH}\end{array}$ & 1.66 & 1.9 & - & - & S9 \\
\hline $\begin{array}{l}\text { 3D N- } \\
\text { graphene }\end{array}$ & $\begin{array}{l}\text { Pyrolysis } \\
\text { of GO with } \\
\text { polypyrrole }\end{array}$ & 0.142 & $\begin{array}{l}0.1 \mathrm{M} \\
\mathrm{KOH}\end{array}$ & 1.62 & $\begin{array}{l}1.9 \text { at } 1.5 \\
\mathrm{~mA} / \mathrm{cm}^{2}\end{array}$ & - & - & S10 \\
\hline $\begin{array}{l}\mathrm{N}, \mathrm{P} \\
\text { codoped } \\
\text { 3D } \\
\text { mesoporo } \\
\text { us C }\end{array}$ & $\begin{array}{l}\text { Polymeriza } \\
\text { tion+Pyrol } \\
\text { ysis }\end{array}$ & 0.5 & $\begin{array}{l}0.1 \mathrm{M} \\
\mathrm{KOH}\end{array}$ & 1.3 & 1.58 & 193 & & S11 \\
\hline $\begin{array}{l}\text { Porous } \\
\text { nanorod } \\
\text { Perovskit } \\
\mathrm{e},\end{array}$ & $\begin{array}{l}\text { Chemical+ } \\
\text { electrospin } \\
+ \text { pyrolysis }\end{array}$ & 0.41 & $\begin{array}{l}0.1 \mathrm{M} \\
\mathrm{KOH}\end{array}$ & 1.53 & 1.75 & - & $\begin{array}{l}81 \% \text { after } \\
3.33 \mathrm{~h}\end{array}$ & S12 \\
\hline
\end{tabular}




\begin{tabular}{|c|c|c|c|c|c|c|c|c|}
\hline $\begin{array}{l}\mathrm{La} 0.5 \mathrm{Sr}_{0.5} \\
\mathrm{Co}_{0.8} \mathrm{Fe}_{0.2} \\
\mathrm{O}_{3} / \mathrm{N}- \\
\mathrm{rGO}\end{array}$ & & & & & & & & \\
\hline $\begin{array}{l}\text { 3D N,S- } \\
\text { rGO } \\
3 \mathrm{D} \\
\mathrm{CoFe}_{2} \mathrm{O}_{4} \\
/ \mathrm{N}, \mathrm{S}-\mathrm{rGO}\end{array}$ & $\begin{array}{l}\text { Chemical+ } \\
\text { hydrotherm } \\
\text { al }\end{array}$ & 1.02 & $\begin{array}{l}0.1 \mathrm{M} \\
\mathrm{KOH} \\
\\
0.1 \mathrm{M} \\
\mathrm{KOH}\end{array}$ & 1.60 & $\begin{array}{l}1.95 \\
1.82\end{array}$ & - & $\begin{array}{l}76 \% \text { after } \\
11.1 \mathrm{~h}\end{array}$ & $\begin{array}{l}\text { S13 } \\
\text { S13 }\end{array}$ \\
\hline $\begin{array}{l}\mathrm{CoO}_{x} / \mathrm{N}- \\
\mathrm{C}\end{array}$ & $\begin{array}{l}\text { Hydrother } \\
\text { mal+pyroly } \\
\text { sis }\end{array}$ & 0.54 & $\begin{array}{l}0.1 \mathrm{M} \\
\mathrm{KOH}\end{array}$ & 1.55 & 1.65 & - & - & S14 \\
\hline $\begin{array}{l}\text { 3D N,S- } \\
\text { graphene } \\
\text { nanoribb } \\
\text { on } \\
\text { network }\end{array}$ & Pyrolysis & 0.3 & $\begin{array}{l}1 \mathrm{M} \\
\mathrm{KOH}\end{array}$ & 1.53 & 1.59 & 47 & $\begin{array}{l}\sim 90 \% \text { after } 24 \\
\mathrm{~h}\end{array}$ & S15 \\
\hline $\begin{array}{l}\mathrm{N}, \mathrm{P}- \\
\mathrm{rGO} / \mathrm{C}\end{array}$ & $\begin{array}{l}\text { Chemical+ } \\
\text { pyrolysis }\end{array}$ & 0.141 & $\begin{array}{l}0.1 \mathrm{M} \\
\mathrm{KOH}\end{array}$ & 1.53 & 1.57 & - & - & S16 \\
\hline $\begin{array}{l}\mathrm{N}, \mathrm{O}- \\
\text { graphene/ } \\
\text { CNT } \\
\text { hydrogel } \\
\text { in 2D } \\
\text { form }\end{array}$ & $\begin{array}{l}\text { Chemical+ } \\
\text { hydrotherm } \\
\text { al }\end{array}$ & 1.75 & $\begin{array}{l}0.1 \mathrm{M} \\
\mathrm{KOH}\end{array}$ & 1.74 & 1.97 & 141 & $\begin{array}{l}\sim 80 \% \text { after } \\
800 \text { potential } \\
\text { cycles }\end{array}$ & S17 \\
\hline $\begin{array}{l}\text { N- } \\
\text { graphene/ } \\
\text { SWCNT } \\
\text { hybrids }\end{array}$ & $\begin{array}{l}\text { Thermal } \\
\text { CVD }\end{array}$ & 5 & $\begin{array}{l}0.1 \mathrm{M} \\
\mathrm{KOH}\end{array}$ & 1.45 & 1.64 & 83 & - & S18 \\
\hline $\begin{array}{l}\text { 3D N- } \\
\text { graphene } \\
\text { scaffold }\end{array}$ & $\begin{array}{l}\text { Chemical+ } \\
\text { hydrotherm } \\
\text { al }\end{array}$ & 10.2 & $\begin{array}{l}0.1 \mathrm{M} \\
\mathrm{KOH}\end{array}$ & 1.65 & 1.82 & 244.9 & - & S19 \\
\hline $\begin{array}{l}\mathrm{Ni}- \\
\mathrm{Co} @ \mathrm{~N}- \\
\mathrm{CNT}\end{array}$ & $\begin{array}{l}\text { Hydrother } \\
\text { mal+pyroly } \\
\text { sis }\end{array}$ & 0.464 & $\begin{array}{l}0.1 \mathrm{M} \\
\mathrm{KOH}\end{array}$ & 1.55 & 1.65 & 177 & $\begin{array}{l}>95 \% \text { after } \\
1000 \text { cycles }\end{array}$ & S20 \\
\hline $\begin{array}{l}\text { Porous } \\
\text { N-carbon } \\
\text { tubes }\end{array}$ & $\begin{array}{l}\text { Chemical+ } \\
\text { hydrotherm } \\
\text { al+pyrolysi } \\
\text { s }\end{array}$ & 0.15 & $\begin{array}{l}0.1 \mathrm{M} \\
\mathrm{KOH}\end{array}$ & 1.92 & 1.97 & - & - & S21 \\
\hline $\begin{array}{l}\text { N-porous } \\
\text { carbon }\end{array}$ & $\begin{array}{l}\text { Hydrother } \\
\text { mal+pyroly } \\
\text { sis }\end{array}$ & 0.2 & $\begin{array}{l}0.1 \mathrm{M} \\
\mathrm{KOH}\end{array}$ & 1.53 & 1.61 & - & - & S22 \\
\hline $\begin{array}{l}\mathrm{N}- \\
\text { MWCNT }\end{array}$ & $\begin{array}{l}\text { Chemical+ } \\
\text { pyrolysis }\end{array}$ & 0.2 & $\begin{array}{l}0.1 \mathrm{M} \\
\mathrm{NaOH}\end{array}$ & 1.54 & 1.63 & 78 & - & S23 \\
\hline
\end{tabular}




\section{* Calculation of mass loading of stacked double layer catalyst on glassy carbon electrode}

The electrode surface area $=0.196 \mathrm{~cm}^{2}$ [commercial glassy carbon electrode of $5 \mathrm{~mm}$ diameter] The maximum density of graphite $(\mathrm{D})=2.266 \mathrm{~g} / \mathrm{cm}^{3}$

The average thickness of G-BNG catalyst (excluding void which was introduced during TEM sample making) $\sim 3 \mathrm{~nm}=3 \times 10^{-7} \mathrm{~cm}$

The average volume of the double later $(\mathrm{G}-\mathrm{BNG})$ catalyst $(\mathrm{V})=0.196 \times 3 \times 10^{-7}=0.588 \times 10^{-7} \mathrm{~cm} 3$

Therefore, the mass of the double layer catalyst on electrode $=\mathrm{DV}=0.133 \times 10^{-6} \mathrm{~g}$

The mass loading onto the electrode surface $=0.68$ microgram $/ \mathrm{cm}^{2}=0.00068 \mathrm{mg} / \mathrm{cm}^{2}$ (this is a maximum value).

\section{References}

(S1) Paul, R.; Voevodin, A. A.; Zemlyanov, D.; Roy, A. K.; Fisher, T. S. Microwave-assisted surface synthesis of a boron-carbon-nitrogen foam and its desorption enthalpy. Adv. Funct. Mater. 2012, 22, 3682-3690.

(S2) Kim, S. Y.; Park, J.; Choi, H. C.; Ahn, J. P.; Hou, J. Q.; Kang, H. S. X-ray photoelectron spectroscopy and first principles calculation of BCN nanotubes. J. Am. Chem. Soc. 2007, 129, 1705-1716.

(S3) Paul, R.; Etacheri, V.; Pol, V. G.; Hu, J.; Fisher, T. S. Highly porous three-dimensional carbon nanotube foam as a freestanding anode for a lithium-ion battery. $R S C A d v$. 2016, 6(83), 7973479744.

(S4) Ferrari, A. C. Raman spectroscopy of graphene and graphite: Disorder, electron-phonon coupling, doping and nonadiabatic effects. Solid State Comm. 2007, 143, 47-57.

(S5) Chabot, V.; Kim, B.; Sloper, B.; Tzoganakis, C.; Yu, A. High yield production and purification of few layer graphene by gum arabic assisted physical sonication. Sci. Rep. 2013, 3, 1378.

(S6) Wang, L.; Yin, F.; Yao, C. N-doped graphene as a bifunctional electrocatalyst for oxygen reduction and oxygen evolution reactions in an alkaline electrolyte. Int. J. Hydrog. Energy 2014, 39, 15913-15919.

(S7) Jiang, Z.; Jiang, Z. J.; Maiyalagan, T.; Manthiram, A. Cobalt oxide-coated N- and B-doped graphene hollow spheres as bifunctional electrocatalysts for oxygen reduction and oxygen evolution reactions. J. Mater. Chem. A 2016, 4, 5877-5889.

(S8) Ganesan, P.; Prabu, M.; Sanetuntikul, J.; Shanmugam, S. Cobalt sulfide nanoparticles grown on nitrogen and sulfur codoped graphene oxide: an efficient electrocatalyst for oxygen reduction and evolution reactions. ACS Catal. 2015, 5, 3625-3637. 
(S9) Cheng, Y.; Tian, Y.; Fan, X.; Liu, J.; Yan, C. Boron doped multi-walled carbon nanotubes as catalysts for oxygen reduction reaction and oxygen evolution reaction in in alkaline media. Electrochimica Acta 2014, 143, 291-296.

(S10) Lin, Z.; Waller, G. H.; Liu, Y.; Liu, M.; Wong, C. P. 3D nitrogen-doped graphene prepared by pyrolysis of graphene oxide with polypyrrole for electrocatalysis of oxygen reduction reaction. Nano Energy 2013, 2, 241-248.

(S11) Zhang, J.; Zhao, Z.; Xia, Z.; Dai, L. A metal-free bifunctional electrocatalyst for oxygen reduction and oxygen evolution reactions. Nat. Nanotechnol. 2015, 10, 444-452.

(S12) Park, H. W.; Lee, D. U.; Zamani, P.; Seo, M. H.; Nazar, L. F.; Chen, Z. Electrospun porous nanorod perovskite oxide/nitrogen-doped graphene composite as a bi-functional catalyst for metal air batteries. Nano Energy 2014, 10, 192-200.

(S13) Yan, W.; Cao, X.; Tian, J.; Jin, C.; Ke, K.; Yang, R. Nitrogen/sulfur dual-doped 3D reduced graphene oxide networks supported $\mathrm{CoFe}_{2} \mathrm{O}_{4}$ with enhanced electrocatalytic activities for oxygen reduction and evolution reactions. Carbon 2016, 99, 195-202.

(S14) Jin, H.; Wang, J.; Su, D.; Wei, Z.; Pang, Z.; Wang, Y. In situ cobalt-cobalt oxide/N-doped carbon hybrids as superior bifunctional electrocatalysts for hydrogen and oxygen evolution. J. Am. Chem. Soc. 2015, 137, 2688-2694.

(S15) Yang, H. B.; Miao, J.; Hung, S. F.; Chen, J.; Tao, H. B.; Wang, X.; Zhang, L.; Chen, R.; Gao, J.; Chen, H. M.; Dai, L.; Liu, B. Identification of catalytic sites for oxygen reduction and oxygen evolution in N-doped graphene materials: Development of highly efficient metal-free bifunctional electrocatalyst. Sci. Adv. 2016, 2, e1501122.

(S16) Li, R.; Wei, Z.; Gou, X. Nitrogen and phosphorus dual-doped graphene/carbon nanosheets as bifunctional electrocatalysts for oxygen reduction and evolution. ACS Catal. 2015, 5, 41334142.

(S17) Chen, S.; Duan, J.; Jaroniec, M.; Qiao, S. Z. Nitrogen and oxygen dual-doped carbon hydrogel film as a substrate-free electrode for highly efficient oxygen evolution reaction. $A d v$. Mater. 2014, 26, 2925-2930.

(S18) Tian, G. L.; Zhao, M. Q.; Yu, D.; Kong, X. Y.; Huang, J. Q.; Zhang, Q.; Wei, F. Nitrogendoped graphene/carbon nanotube hybrids: in situ formation on bifunctional catalysts and their superior electrocatalytic activity for oxygen evolution/reduction reaction. Small 2014, 10(11), 2251-2259.

(S19) Chen, S.; Duan, J.; Ran, J.; Jaroniecb, M.; Qiao, S. Z. N-doped graphene film-confined nickel nanoparticles as a highly efficient three-dimensional oxygen evolution electrocatalyst. Energy Environ. Sci. 2013, 6, 3693-3699.

(S20) Yu, J.; Zhong, Y.; Zhou, W.; Shao, Z. Facile synthesis of nitrogen-doped carbon nanotubes encapsulating nickel cobalt alloys 3D networks for oxygen evolution reaction in an alkaline solution. J. Power Sources 2017, 338, 26-33. 
(S21) Pan, T.; Liu, H.; Ren, G.; Li, Y.; Lu, X.; Zhu, Y. Metal-free porous nitrogen-doped carbon nanotubes for enhanced oxygen reduction and evolution reactions. Sci. Bull. 2016, 61(11), 889896.

(S22) Zhao, Y.; Nakamura, R.; Kamiya, K.; Nakanishi, S.; Hashimoto, K. Nitrogen-doped carbon nanomaterials as non-metal electrocatalysts for water oxidation. Nat. Commun. 2013, 4, 2390.

(S23) Davodi, F.; Tavakkoli, M.; Lahtinen, J.; Kallio, T. Straightforward synthesis of nitrogendoped carbon nanotubes as highly active bifunctional electrocatalysts for full water splitting. $J$. Catal. 2017, 353, 19-27. 